\title{
The Smile Aesthetics and its Implications in Social Prosthetic Rehabilitations: A Case Report
}

\author{
Magda Ecaterina Antohe*1, Cristina Iordache*1 and Gabriel Drug ${ }^{2}$ \\ ${ }^{1}$ Faculty of Dentistry, Grigore T. Popa University of Medicine and Pharmacy, Romania \\ ${ }^{2}$ Bioinginer, Country Hospital, Bacau, Romania
}

Received: November 29, 2017; Published: December 06, 2017

*Corresponding author: Cristina Iordache, Lecturer, Phd, Specialist in general dentistry, Specialist in dento-alveolar surgery, Faculty of Dental Medecine, Grigore T Popa, University of Medecine and Pharmacy, Iasi, Romania, Tel: 0040745503852; Email: ccmiiordache@yahoo.com

\begin{abstract}
The aim of this study is to individualize the correlative aspects between the type of smile, the degree of visibility of the teeth and the way in which the chosen therapeutic solution, in accordance with the particularity of the clinical case, offers the ultimate clinical success in terms of aesthetic requirements.In order to make the aesthetic evaluations and the contour matches we used photos for the patients faces and teeth and we processed these photos in Corel Draw 7.0. We manually traced the contour of the face and the frontal tooth and we matched the two shapes changing accordingly their dimensions; the smile type was also established through contour detection.The type of prosthesis is fully in agreement with the particularity of the prosthetic field, the presence or absence of specific prosthetic preparation corroborated with the aesthetic restoration through individualized modeling decisively influences the final results.
\end{abstract}

Keywords: Clinical Case; Removable Prosheses; Smile; Aesthetic Evaluation

\section{Introduction}

The multitude of clinical cases requiring aesthetic therapy anchored in both the fixed prosthesis registry and the movable prosthesis, makes it impossible to classify the treatment according to the state of edification [1-4]. What can be deduced from a thorough clinical examination is the extent of the edentation, the degree of compensation in the odontal, periodontal, mucosal and bone support, the maintenance of the stable occlusion relationship and the type of smile [5-7]. The smile involves altering the physiognomy in all the facial stages, but the anatomy of the lower floor level is most important: the labyrinth extends, the upper lip rises and stretches, and the free edge of the lower lip becomes concave [812]. A distance can be measured between the mouth commisura; the smile index can also be calculated: the ratio between the vertical and horizontal dimensions of the laughing slit associated with the smile. The amplitude of the smile determines the degree of exposure of the dental arches and thereby the definition of the area of aesthetic interest to be taken into account during the dental arcade restoration treatments. Smile is a form of non-verbal communication, most often associated with the exposure of dental arches. Appreciation of the relationships between upper lip, lower lip, and mouth commisure with the dental arches during smile is one of the important stages of examination in dental aesthetics [1315].
Aim: The aim of this study is to individualize the correlative aspects between the type of smile, the degree of visibility of the teeth and the way in which the chosen therapeutic solution, in accordance with the particularity of the clinical case, offers the ultimate clinical success in terms of aesthetic requirements.

\section{Material and Methods}

In order to make the aesthetic evaluations and the contour matches we used photos for the patient's faces and teeth and we processed these photos in Corel Draw 7.0. We manually traced the contour of the face and the frontal tooth and we matched the two shapes changing accordingly their dimensions; the smile type was also established through contour detection. We preferred to perform these procedures by hand because the automated functions for contour tracing do not deal correctly with the anatomical particular details.

\section{Results and Discussion}

The clinical presentation shown here is representative of a social approach, in which we pay special attention to the aestheticfunctional binomial which governs the current therapies, in these particular situations the socio-economic criteria are restrictive. The 55-year-old PZ patient following the clinical and paraclinical 
assessment was diagnosed as follows: General condition affected by the HTA stage offset, under medication, that favours the treatment stages; Chronic superficial periodontitis at the level of the anterior frontal teeth, of a plurifactorial etiology with slow progression, favorable prognosis by treatment, currently untreated, Total maxillary edentation of plurifactorial aetiology, which induces functional, masticatory, physiological, phonetic, swallowing disorders, evolving slowly, giving local complications such as edema and atrophy of the edentated and loco-regional crest with articular dysfunction, with prognosis favorably untreated, Kennedy Class I stretched mandibular partial joint jaw, of mixed etiology, which induces functional discomfort, swallowing, physiognomy, slowly evolving, causing local complications such as resorption and atrophy of the edentar ridge localized and loco-regional disorders such as joint dysfunction, prognosis favoring treatment, mixed prosthesis treated at this stage.

Malocclusion by modifying occlusal morphological parameters, asymmetric occlusion curves, irregular oblique occlusion; Moderate joint dysfunction, with etiology extended stretch condition and reduced untreated partial edentary states and other detectable etiology through paraclinical examinations, giving discomfort to the masticatory, slowly evolving, with prognosis favorably through treatment Extrapostural and eccentric mandibulo-cranial malrelation, having the etiology of the partialextended edentation and the partially reduced untreated anterior edema, leading to masticatory, swallowing disorders, deglutition, slowly evolving, with favorable prognosis through treatment; Assessment of clinical and biological indices is a decisive starting point for the development of a therapeutic plan. The positive aspects characterizing the general clinical-biological indices are represented by the general good condition of the patient, which allows the development of the treatment stages.The loco-regional indices are characterized by negative aspects negative positioned at the TMJ level, noting aspects of mandibular dynamics characterized by asymmetric condylous excursions, which accompany left-sided latency. Regarding the local odonto-periodontal clinical-biological indices there is a small number of odonto-periodontal units, and the muco-bone support is characterized by the presence of resilient mucosa, irregular crests, negative indices that can be posed by specific training or the choice of a biomaterial with a structure adapted to these particularities (Figure 1).

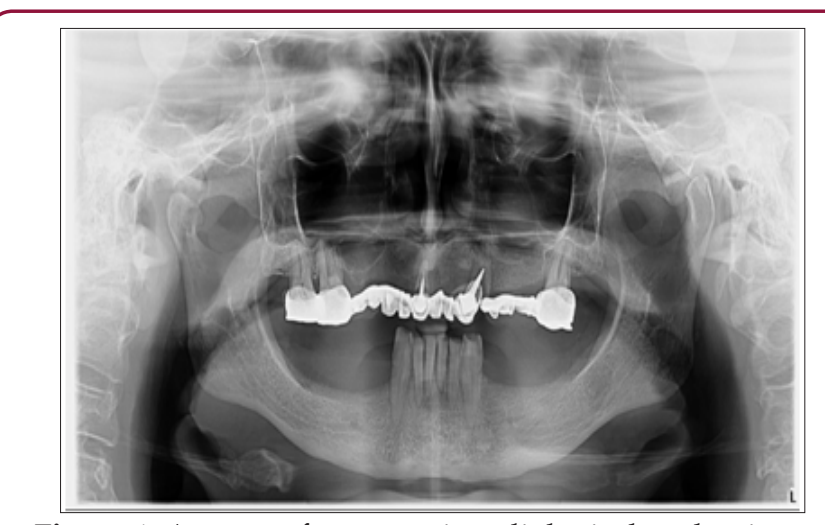

Figure 1: Aspects of panoramic radiological evaluation.
The negative aspects related to occlusion reside in the changing of the static occlusion parameters due to the morphology and functionality of the mixed restorations which the patient presented, inducing changes in the dynamic occlusion trajectories. The therapeutic plan was governed by the following objectives:

a. Prophylaxis objective:nonspecific local prophylaxissanitary education, general prophylaxis - vitamin therapy, psychotherapy ,specific local prophylaxis.

b. Curative objective: morphological: the maxillary achievement of a complete denture restoration and at the mandibular level of some restorations fixed and partially removable flexible prostheses, restoring the dento-alveolar integrity.

c. Functional: restoring the functions of the stomatognate system: mastication, phonation, swallowing, physiognomy.

At the mandible we face a balanced symmetric-asymmetric edification, corroborated with the orientation of the vector RP(resistance-pressure) towards posterior, shows us both the deficient support area and the topography of the elements of maintenance, support and stabilization, if we remain as a variant in the territory of the removable prosthesis. After investigating and analyzing the treatment plan according to all the mentioned principles and criteria, we chose the following therapeutic solution: at the maxilla-complete acrylic denture, the teeth were chosen from the composite, the shape of the teeth being fully aligned with the shape of the face and the architecture of the maxilla prosthetic field. Regarding the mandibulary solution,we chose an mixed prosthesis variant composed:fixed metal-ceramic restoration at levels 33, $32,31,41,42,43,44$ and partially removable flexible prosthesis consisting of a dentum-mucosal plaque connector, 2 metal-acrylic mixed sutures with 8 artificial teeth, acrylic, anatomorphic, mediocuspidate and support, stabilization and support elements: elastic clasps. The stages of the treatment:

Preparation of the body and oral cavity

a) Health education,

b) General training,

c) Preprotetic (non-specific) preparation,

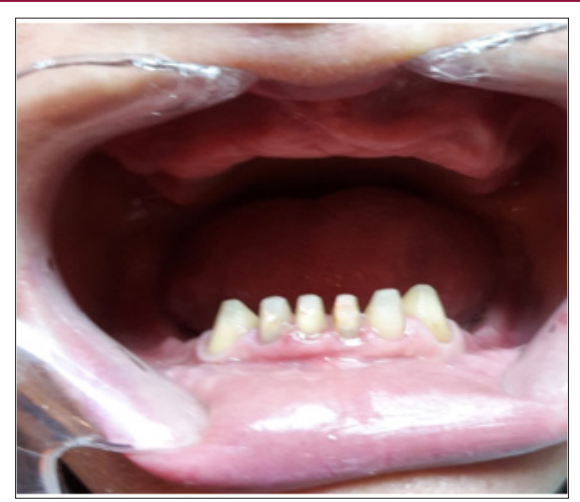

Figure 2: Aspect ofpreparation of the teeth at the mandibular level. 
d) Preprotetic (specific) preparation-Recovery of organic substructures and correction of preparations at mandibular level (Figure 2).

The proper prosthetic treatment concerned the following steps:

a) Preliminaryimpression recorded in alginic material.

b) Functional impression-it is important to note that on the functional model, all the hybrid prosthesis, both the fixed and the mobilizable component, were made for the purpose of full harmony morpho-functional between the two components. The functional fingerprint was recorded with addition silicon.

c) The recording of the mandibular-cranial relations with the help of the occlusion models and the transfer on the simulator.

d) The checking of the prostheses in the wax. This step is important and precise by superimposing the therapeutic decision marks over the resulting technological elements.

e) Clinical examination, adaptation and fixation by temporary cementation of the fixed prosthesis and verification, adaptation of the partial removable prosthesis (Figure 3).

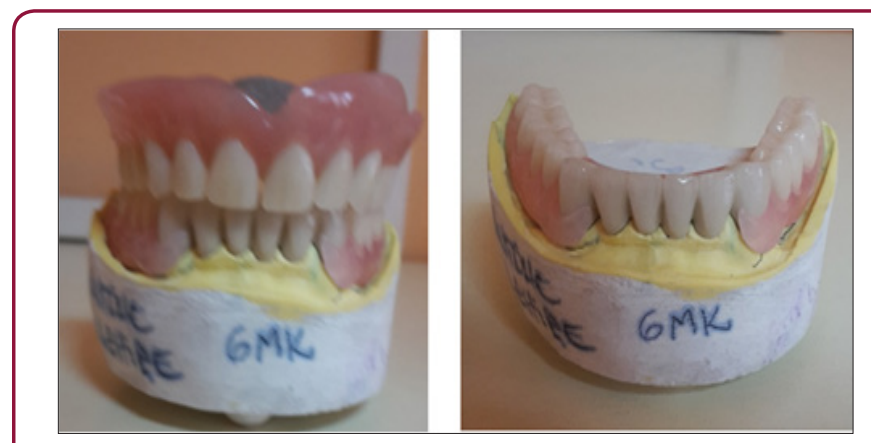

Figure 3: Aspects of maxillarycomplete denture andpartially mandibular removable.

f) Fixation by permanent cementing of the fixed prothesis.

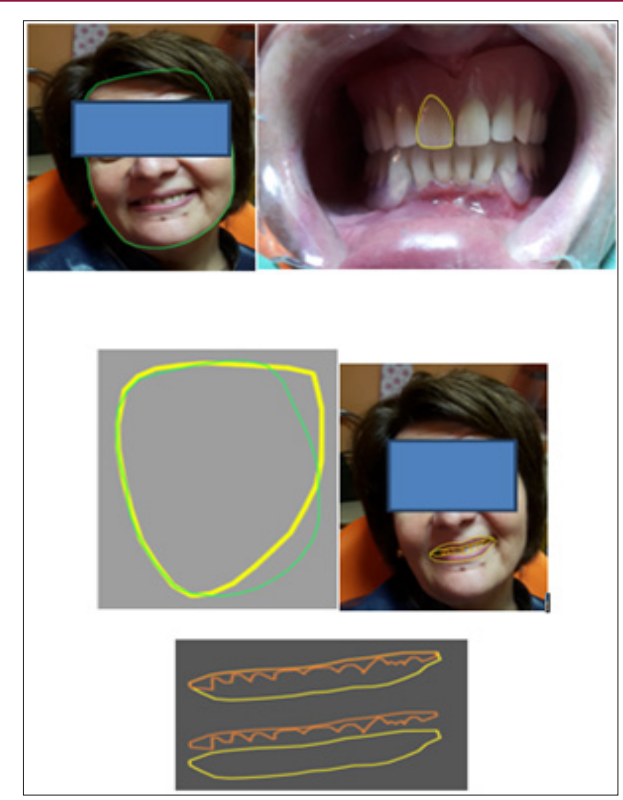

Figure 4: Superposability of the shape of the face and of the dental shape. prostheses and fixed componentent. After superposing the dental contour on the facial one, an optimsuperposing results, and the framing of the smile in the convex one, with contact restores aesthetics of the patient. The final results of the clinical case offer the image of the rehabilitation edentatotal or partial stretched by total prosthesis, respectively mandibular joint prosthesis, therapeutic solutions that created the cranio-mandibular reposition, as well as the restoration of all the functions affected by edentation (Figure 4). A very important factor in obtaining a result of high-level prosthetic treatment is the harmony between the shape of the teeth, their position, their color and the appearance of the gingival tissues adjacent to them, because a harmonious ensemble can not be defined only under conditions of free periodontal structures.For this reason, the gingival color, the level and extent of exposure of the free gum and the interdental papillae in the smile, even the aspect of the fibroma that covers the alveolar processes, are elements whose analysis completes the examination of dento-facial aesthetics.

\section{Conclusion}

a) Aesthetic exigency, which has become a social phenomenon in today's life, represents for contemporary dental medicine not only as an important dental problem but also a professional duty.

b) The type of prosthesis is fully in agreement with the particularity of the prosthetic field, the presence or absence of specific prosthetic preparation corroborated with the aesthetic restoration through individualized modeling decisively influences the final results.

c) It is especially important that the therapeutic solution chosen in full accord with the degree of vision of the teeth and the type of smile giving the patients' natural, but also the elegant result of the precision and observance of the clinicalbiological parameters that required the rehabilitation.

\section{References}

1. Waliszewski M (2005) Restoring dentate appearance: a literature review for modern complete denture esthetics. J Prosthet Dent 93(4): 386-394.

2. Forna N (2011) Proshetic Denture. Enciclopedica, Bucharest, Romania.

3. Ifteni G, Apostu A, Ioanid N (2017) A clinical evaluation of fixed dental prostheses without regular maintenance. Romanian Journal of Oral Rehabilitation 9(2): 49-53.

4. Perea C, Suárez-García MJ, Del Río J, Torres-Lagares D, Montero J, et al. (2013) Oral health-related quality of life in complete denture wearers depending on their socio-demographic background, prosthetic-related factors and clinical condition. Med Oral Patol Oral Cir Bucal 18(3): 371380 .

5. Montero J, Macedo C, López-Valverde A, Bravo M (2012) Validation of the oral health impact profile (OHIP-20sp) for Spanish edentulous patients. Med Oral Patol Oral Cir Bucal 17(3): 469-476.

6. Slade GD, Spencer AJ (1994) Development and evaluation of the oral health impact profile. Community Dent Health 11(1): 3-11.

7. Wong AH, Cheung CS, Mc Grath C (2007) Developing a short form of Oral Health Impact Profile (OHIP) for dental aesthetics: OHIP-aesthetic. Community Dent Oral Epidemiol 35(1): 64-72.

8. Montero-Martín J, Bravo-Pérez M, Albaladejo-Martínez A, HernándezMartín LA, Rosel-Gallardo EM (2009) Validation the Oral Health Impact 
Profile (OHIP-14sp) for adults in Spain. Med Oral Patol Oral Cir Bucal 14(1): 44-50.

9. Pan S, Awad M, Thomason JM, Dufresne E, Kobayashi T (2008) Sex differences in denture satisfaction. J Dent 36(5): 301-308.

10. Mehl C, Kern M, Freitag-Wolf S, Wolfart M, Brunzel S, et al. (2009) Does the oral health impact profile questionnaire measure dental appearance? Int J Prosthodont 22(1): 87-93.

11. Ide R, Hoshuyama T, Wilson D, Takahashi K, Higashi T (2006) Association of psychological well-being with oral conditions in Japanese workers. J Occup Health 48(6): 487-493.
12. Wolfart S, Quaas AC, Freitag S, Kropp P, Gerber WD, et al. (2006) Subjective and objective perception of upper incisors. J Oral Rehabil 33(7): 489-495.

13. Fani M, Farmani S, Bagheri R (2015) Fracture Toughness of Resin Composites under Different Modes and Media. Review of Articles. J Dent Biomater 2(3): 73-82.

14. Mitra SB, Wu D, Holmes BN (2003) An application of nanotechnology in advanced dental materials. J Am Dent Assoc 134(10): 1382-1390.

15. Desai R, Durham J, Wassell RW, Preshaw PM (2014) Does the mode of administration of the Oral Health Impact Profile-4 affect the outcome score? J Dent 42(1): 84-89.

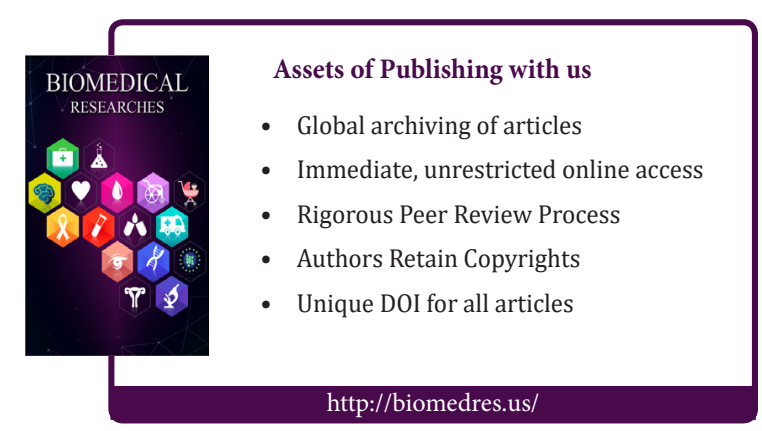

